\title{
Research on the Structure and Measurement of Communication Capabilities of Enterprise Managers
}

\author{
Taibo Jiang \\ The open university of Zhongshan, Zhongshan, Guangdong, 528403
}

Keywords: structure and measurement; communication capability; enterprise managers

\begin{abstract}
Interpersonal communication skills are one of the important qualities that modern enterprise managers must possess. With the integration of the world economy today, with the interpenetration of members of different cultures in the organization, the human communication capabilities of managers have become an important factor influencing management performance. It is of great significance to study the interpersonal communication capabilities of enterprise managers. Interpersonal communication skills refer to the ability to communicate effectively with individuals and in appropriate ways in social interaction. Effectiveness is the extent to which communication behaviors contribute to individual goals and the realization of relationship goals. The degree of appropriateness is consistent with the degree of situational and relationship constraints. Effectiveness and appropriateness are important in assessing the level of accessibility.
\end{abstract}

\section{Introduction}

Gödel's theorem proves that a sufficiently abundant and coherent theory cannot prove its non-contradiction by itself, or by less imperfect or more "weak" means than its own; a theoretical system if only Its own means for the tool to prove itself, it will certainly lead to some proposition that can not determine its true and false. Therefore, any theoretical system is always incomplete on its own. If a theoretical system is to prove its non-contradiction, it must rely on another theory that is more complete or more "stronger" than it. 'System theory is a product of highly developed science. It provides us with a scientific way of thinking that fits the characteristics of a complex system and has important guiding value for understanding complex systems. The brain that carries mental activity is an open and complex self-organization. The system is an indisputable fact, and communication, as an important psychological activity for information exchange between people and the outside world, is also a complex self-organizing system with dynamic and open system characteristics. Only a systematic thinking can build a scientific communication ability structure.

\section{Quantification Study Communication Ability of Enterprise managers}

A good measure is the standard for checking the quality of the interstitials. An ideal questionnaire excludes reliability (validity) and effectiveness (validity) as indicators. Confidence is the proportion of the variance of the true scores in the questionnaire children among the total variance. Validity is the ratio of the variance of the scores related to the measurement objectives to the total variance, including construct validity, content validity, compatibility validity, and so on. A good questionnaire should also have a certain degree of discrimination, discriminating ability, and be able to identify the level of human ability. A reliable and effective questionnaire is the best proof of the rationality and effectiveness of the theoretical concept. To this end, the reliability and validity of the questionnaire were tested in many aspects. Validity tests included structural validity and The analysis of tolerance, project differentiation and discriminating power, reliability testing mainly carried out Cronbach's internal consistency reliability coefficient test. It should be noted that when the structural validity test (see empirical study 2) was performed, four items with high degree of commonality between the two factors were deleted, and the following analysis was performed on the remaining 27 items. The content validity of the questionnaire examines whether the measurement item can reflect the content or subject to be measured. The total correlation method is 
used to calculate the correlation between the score of each item and the total score. The correlation coefficient of the fruit is not significant, indicating that Project differentiation is low. The more significant the correlation coefficient, the higher the discriminant validity. The empirical results show that the correlations between the items of the interpersonal communication ability and the sum totals of the subscales are all significant at the level of the instrument guilt, and there are two questions for the total correlation between the items in the .02 any blade .29. There are 6 questions between .0 .30 and $0 . .39$, and there are questions above .040 , indicating that the discriminant validity of the interpersonal communication ability scale is good. Interpersonal communication skills Each item has a score of 0.20 or less, 1 of 0.20 to 0.29 , 11 of 0.30 to 0.39 , and 3 of .040 or higher. The title indicates that each item has a good discriminating power.

In order to test the discriminatory power of the questionnaire measurement on managers and employees, this study analyzed the variance of the communication skills of 119 employees and the third-level managers. The homogeneity test of variance showed that the participants were from different populations (interpersonal communication skills, management communication). The variance consistency test results of ability and communication ability were significant at $0.54,0.004$, and 0.012, respectively. Therefore, the data was first logarithmically transformed in this study, followed by single factor independent sample variance analysis. The one-way analysis of variance in the ability of managers and employees to measure communication shows that there are very significant differences in the communication skills between employees and three levels of managers. Among them, employees' interpersonal communication skills are generally higher than those of managers. High to low rankings are employees, middle management, senior management, and grassroots managers; while employees' management and communication skills are generally lower than those of managers, and there are significant differences. Among them, the grass-roots managers have the strongest management and communication skills, followed by middle-level managers, senior managers, and employees in descending order; among the three-level managers, there are significant differences in interpersonal communication skills between grass-roots and middle-level managers. Differences did not show significant differences, indicating that there was no significant difference in the communication skills among managers. In general, the questionnaire has a clear identification of employees and managers in the ability to identify management and communication, and also shows differences between employees and managers in interpersonal communication skills.

\section{Study on the Structure of Communication Ability of Enterprise Managers}

This study constructs a management communication capability structure from the perspective of management communication. According to E. Tolman and W. Stephenson, the function of managing communication at the organizational level can be subdivided into emotional function, coordination function, and decision function. Correspondingly, this study divided management communication capabilities into three components: emotional communication ability, coordination communication ability, and decision-making communication ability. This theoretical assumption was confirmed in the measurement of the management communication skills questionnaire developed in this study. In the study of management communication ability prediction, this study selected three factors from the results of the first-order exploratory factor analysis of the questionnaire, which reflected the factors of emotional communication, coordinated communication, and innovation and motivation. These three factors can explain $44.340 \%$ of the total variance, indicating the existence of three components of management communication skills. The meanings of various components of management communication skills are as follows: Innovation Incentive Communication Ability: The senior with high scores is good at inspiring employees to think from multiple angles in the chairing meeting, encouraging new ideas at the meeting, being sensitive to new information, and being tolerant to accepting themselves. different opinions. Emotional communication ability: When the high scorer is dealing with crisis situations, erroneous situations, and interest dispute situations, he can take into account the face of others and be good at using employees to participate in decision-making and other means to mobilize the enthusiasm of subordinates and encourage their 
self-esteem and self-worth. Coordinating and Communicating Skills: High scorers are good at using various communication strategies when dealing with conflicts of interpersonal relationships, conflicts of employment, conflict of goals, and conflicts of interest. They harmonize relationships and powerful conflicts without harming their feelings and achieve consensus. . Communication skills are the performance skills of communication, including the skills to receive and send information. Through exploratory factor analysis and confirmatory factor analysis, three skills traits-verbal expression skills, nonverbal expression skills, and listening skills were selected. Non-verbal expression skills: High scorers indicate that in communicating with people, they use gesture language to express their feelings, their eyes, gestures, and gestures are appropriate; they are dressed appropriately; they can express their meanings in voice and intonation; they use spatial distance to express intimacy. Speech expression skills: High scorers are good at expressing thoughts and feelings, and choosing words accurately and appropriately. Listening ability: High scorers are good at using queries, repeating conversations with others, and making body language such as facing the speaker forward to help clarify the meaning of other people's speech and encourage others to speak. Communication and Cognitive Ability: High scorers have high awareness of themselves, others, and situations in communication, know their own communication goals, be good at seeing other people's thoughts and feelings (empathy), and know their actions at the time, Appropriate performance on the occasion. It is the ability to accurately understand and correctly judge the self, other people, and communication situations in the communication process. Communicative tendencies are the driving forces of communication preferences and behaviors, including: motivation for communication: people with higher scores desire to understand and be understood, and have the desire to influence others through communication. Communication anxiety: High scorers are more anxious in their speeches. The three components belong to different sides of the same level of psychology.

\section{Research on the Managerial Communication Ability Conversion Mechanism}

On the one hand, the ability to communicate and understand helps improve communication skills. Communicative cognitive schemas are a high-level summary of skill manipulation methods and their effects. Good communication is based on effectiveness and appropriateness. Effective communication requires a clear understanding of your communication goals. Appropriate communication requires communicators to have a clear understanding of themselves, others, and situations in order to determine what Time, what kind of occasion, what kind of person, and in what way to communicate, in order to achieve the purpose of communication through effective and appropriate means of communication. How to express themselves and what kind of behavioral responses in the context of communication depends largely on his knowledge of people and things, and of course, on other factors such as his communication habits and personality. On the other hand, high-level communication skills tend to reduce people's ability to communicate and understand. A possible explanation is that people are accustomed to using their own skilled behaviors without having to think too much, or even thinking about them to solve problems. This skill of communication skills lowers people's requirements for thinking about events and reduces them. People's cognitive level; Another explanation is that high levels of communication skills will reduce the degree of communication anxiety and psychological stress, that is, reduce the desire for cognition and put people in a state of emboldening. On the one hand, high levels of communication and cognition tend to increase the degree of communication motivation and communication anxiety. Motivation is the result of cognitive imbalances, which can be explained by Piaget's theory of cognitive equilibrium. Piaget believes that cognitive imbalances are the driving force for cognitive activities. When external stimuli cannot be better assimilated by people, they will cause cognitive imbalances and make people feel nervous, and people's purpose of survival lies in To maintain the balance of the body, balance is the basic condition for the existence of the body. The higher the level of people's communication and cognition, the more accurate the judgment of oneself and others, and the easier it is to realize the information asymmetry between themselves and others (distance between information and viewpoints), or even conflicts. The greater the possibility of 
anxiety, the stronger the desire to communicate; the lower the level of awareness, the more blurred is the perception of oneself and others, and the less aware of the difference in mutual understanding, the easier it is to satisfy, which also weakens. Understand the motivation of others and others to understand. On the other hand, communication tends to promote correct cognitive judgments. As a motivational mechanism, communicative tendency provides a driving force for communicative cognition, mobilizes people's positivity in cognition, and thus raises the level of cognitive ability.

There is only an indirect interaction between communication trends and communication skills. This indirect effect is achieved through communication and understanding. Performance in two aspects: high degree of communicative inclination tends to improve the level of communication and cognitive skills, resulting in improved communication skills; low motivation and low anxiety tend to reduce the level of communication and cognitive skills while reducing communication skills. On the other hand, high-level communication skills tend to reduce the level of communication and cognition, thereby reducing the motivation and anxiety of communication; low-level communication skills tend to improve the level of communication and cognition, leading to high communication motivation and communication anxiety.

\section{Conclusion}

The research results confirm the hypothesis that multi-level and multi-dimensional structure of corporate managers' communication skills. That is, the communication capabilities of business managers are composed of two levels, ranging from high to low: management communication and interpersonal communication skills; management and communication skills are composed of emotional communication ability, coordination and communication ability, and innovation and motivation communication; interpersonal communication skills are communicated by Propensity, communication, cognition, and communication skills are three components. Management communication skills have a regulatory effect on the various components of interpersonal communication skills; in the components of interpersonal communication skills, there are two-way mutual influences between communication and cognitive abilities and communication propensities, and communication skills. There is no indication between communication propensities and communication skills. The direct influence is through the indirect influence of communication and cognition. Communication skills are the open doors of the system. Communication tends to provide communication power. Management and communication skills and communication and cognition capabilities play an important synergistic role in the entire structure. Components within the multi-dimensional and multi-layered structure are mutually restricted and cooperate with each other to form a self-organizing system.

\section{References}

[1] Feng Qi. The focus and focus of contemporary management psychology research [J]. School, 2013 (34): 18.

[2] Wang Bolin. Research on Enterprise Human Resource Management Mode Based on Strategy[J]. Research Institute of Management, 2014(6):46.

[3] Wang Limin. Application of Resolving Remuneration System in Enterprise Human Resource Management[J]. 2014(3):76.

[4] Hu Yuhong. Application of Performance Management in Enterprise Human Resource Management [J]. Chinese Economics and Trade, 2014(11): 93-94.

[5] Yang Hongjuan, Wang Le. Labor relationship management in enterprise human resources management[J]. Economic Space Management, 2014(7):132-133. 